\title{
Methodology for Studying Nanoscale Details of Focused Ion Beam Gas-Assisted Etching and Deposition by TEM and Numerical Modeling.
}

\author{
Valery Ray ${ }^{1,4}$, Eddie Chang ${ }^{2}$, Kevin Toula $^{3}$, Sz-Chian Liou$^{1}$, and Wen-An Chiou ${ }^{1}$ \\ 1. NISP Lab, NanoCenter, University of Maryland, College Park, MD 20742, USA \\ 2. Department of Physics, University of Maryland, College Park, MD 20742, USA \\ 3. Department of Material Science and Engineering, University of Maryland, College Park, MD USA \\ 4. PBS\&T, MEO Engineering Co., Inc. Methuen, MA 01844, USA
}

Understanding nanoscale details of Focused Ion Beam (FIB) sputtering, Gas-Assisted Etching (GAE), and beam-induced deposition is critical for a wide range of applications, from patterning thin films to prototyping integrated circuits. Most of research in the field of beam-induced chemistry was historically based on macroscale experiments and observations [1]. While recently developed methodology for investigating details of FIB GAE within a single beam profile [2] relies on analysis of primary ion beam current density profile using multiple HRTEM lattice images, it is difficult for practical routine when resources are limited. This study presents a simplified approach for first-approximation reconstruction of the ion beam characteristics from information available on a single TEM micrograph, and describing nanoscale details of FIB sputtering, Ga implantation, and beam-induced deposition observed during analysis of single-line etching and deposition experiments.

Etching and deposition was carried out in a 30kV Helios $660 \mathrm{FIB} / \mathrm{SEM}$ on fused silica $\left(\mathrm{SiO}_{2}\right)$ substrate coated by $\sim 24 \mathrm{~nm}$ of evaporated Al to prevent charging. Three series of straight lines were made with identical ion beam parameters: first etched by physical sputtering, second etched with $\mathrm{XeF}_{2}$ precursor, and third deposited from $\mathrm{W}$ precursor. Ion dose for lines within each series was set to $0.2 \mathrm{nC} / \mu \mathrm{m}^{2}$, $0.6 \mathrm{nC} / \mu \mathrm{m}^{2}$, and $1.8 \mathrm{nC} / \mu \mathrm{m}^{2}$ and repeated for ion beam currents of $1 \mathrm{pA}, 7.7 \mathrm{pA}$, and $24 \mathrm{pA}$ with dwell of $25 \mathrm{nSec}$ and overlap of $-20 \%$. Lines created by etching and deposition were protected by a layer of ebeam deposited Pt, followed by FIB Pt deposition. TEM lamella positioned across etched lines was prepared by FIB, and FIB cross-section was made across the lines deposited from W precursor.

TEM micrographs of single-line etching profiles were acquired on a JEOL $2100 \mathrm{LaB}_{6}$ TEM and revealed nanoscale details of sputtering (Fig. 1). Ion implantation into $\mathrm{SiO}_{2}$ substrate and agglomeration of the implanted atoms with formation of distinct variable-density zones can be observed within the implanted layer. Lines etched with GAE exhibited dramatic reduction of the apparent implantation and enhanced etching in peripheral areas. Cross-sectional SEM imaging of single-line deposition series not only reveals the fine details of FIB deposition process, but also allows direct observation of Ga damage under the deposited layer. Observations of cross-section images suggest that apparent damage of the substrate material by primary ion beam may continue even after the initial deposition layer has formed. Further study of FIB deposition dynamics using TEM is needed to investigate possible defects and migration of substrate material during the deposition.

Effective diameter and current density distribution within focused ion beam are important characteristics for evaluating beam-induced processes. This information, however, is scarcely available from OEMs of FIB equipment; a practical method for deducing beam characteristics is needed. For practical purposes effective beam diameter is assumed equivalent to the largest width of Ga damage profile measured on corresponding TEM micrographs. To simplify beam current profile, the low-current-density linear beam 
tails were disregarded and bi-Gaussian beam containing wide beam tails with low current density, and a main peak with relatively high current density was modelled. Calculation of the substrate material sputtering assumed that etching profiles with aspect ratio of depth to width $\leq 3: 1$ have linear dependency of depth on the ion dose.

To concurrently simulate implantation and low-aspect-ratio sputtering MATLAB scripts were developed and integrated with SRIM Supporting Software Module (SSSM) and Transport of Ions in Matter (TRIM) packages. Additional MATLAB scripts were created to visualize simulated implantation and etching for comparison with experimental profiles (Fig. 2). Series of beam profile simulations with varied parameters were carried out, and characteristics resulting in simulated profiles with the closest match to experiment were assumed to describe the primary beam. The results showed that implantation of $\mathrm{Ga}$ is lowest and efficiency of FIB GAE is highest in the low-current-density areas of the beam. This in turn suggests that ion beams with current density that is significantly lower than the current Ga FIB technology would be optimal for GAE processes.

\section{References:}

[1] Ivo Utke et al, JVST B 26(4) (2008) p. 1197.

[2] Shida Tan et al, JVST B 30(6) (2012) p. 06F606.

[3] Research was partially supported by NSF-MRSEC (DMR 05-20471) and UMD. Oleg Sidorov of FEI contributed with outstanding execution of single-line etching and deposition design of experiment.
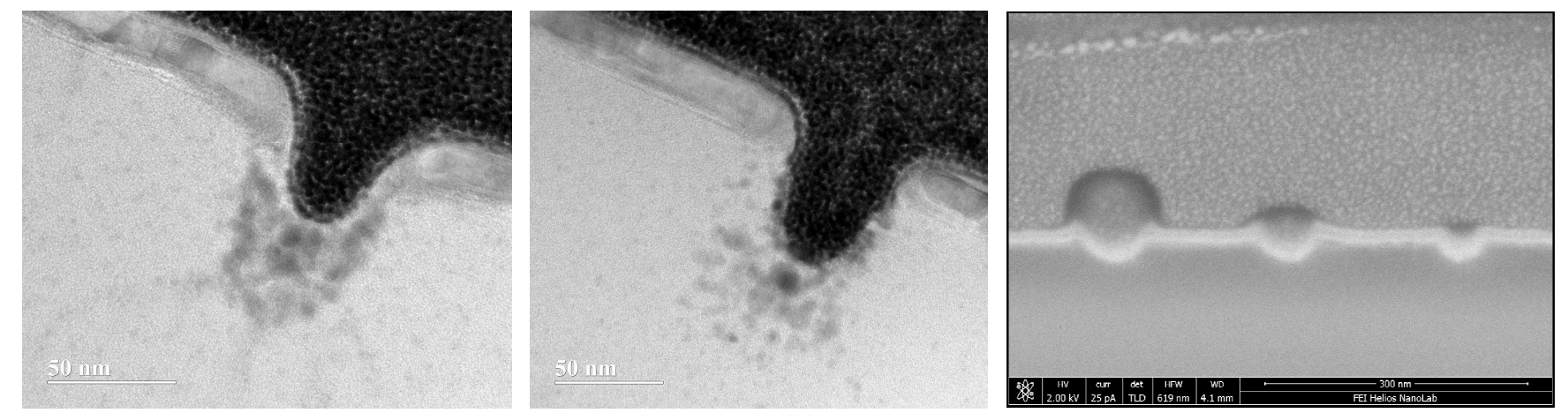

Figure 1. TEM micrographs show profile of physical sputtering and GAE by XeF2 using 7.7pA 30kV $\mathrm{Ga}^{+}$FIB. Cross-sectional SEM image depicts $\mathrm{W}$ deposition with the same primary ion beam.
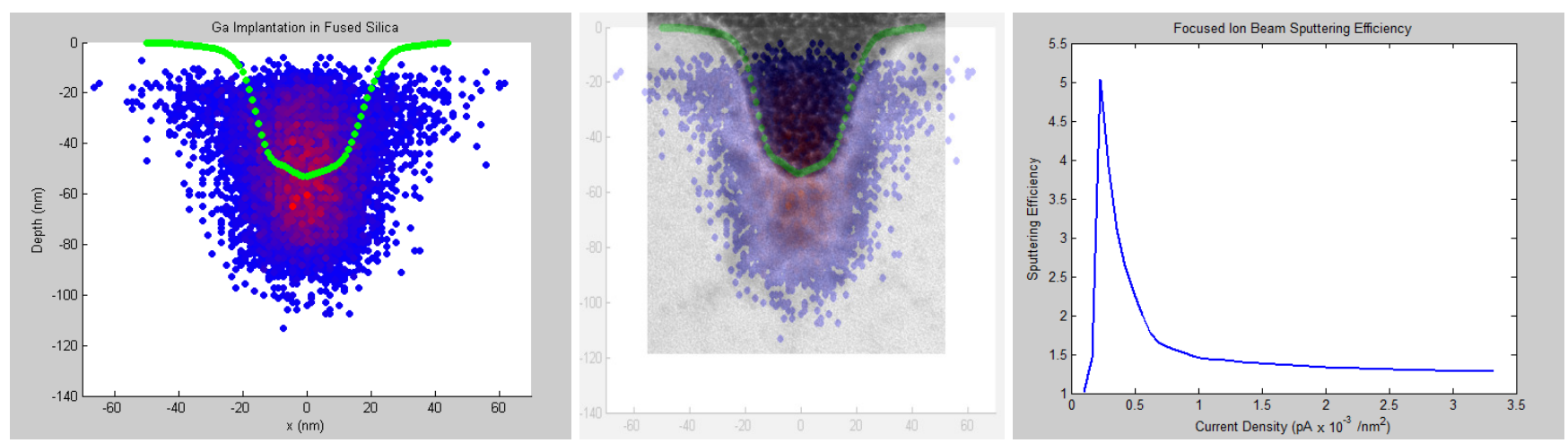

Figure 2. Simulated implantation and sputtering profile compared with matched to scale TEM micrograph, and plot of etching efficiency as function of current density for $7.7 \mathrm{pA} 30 \mathrm{kV} \mathrm{Ga}{ }^{+}$FIB 